\title{
Prognostic Value of Adrenomedullin and Natriuretic Peptides in Uroseptic Patients Induced by Ureteroscopy
}

\author{
Wei Hu, ${ }^{1}$ Pang-hu Zhou, ${ }^{2}$ Wei Wang, ${ }^{1}$ Lijun Zhang, ${ }^{1}$ and Xiao-bin Zhang ${ }^{1}$ \\ ${ }^{1}$ Department of Urology, Renmin Hospital of Wuhan University, No. 238 Liberation Road, Wuhan, Hubei 430060, China \\ ${ }^{2}$ Department of Orthopedics, Renmin Hospital of Wuhan University, No. 238 Liberation Road, Wuhan, Hubei 430060, China \\ Correspondence should be addressed to Pang-hu Zhou; zhoupanghu@126.com
}

Received 28 August 2015; Revised 21 November 2015; Accepted 25 November 2015

Academic Editor: Emanuela Turillazzi

Copyright (c) 2016 Wei Hu et al. This is an open access article distributed under the Creative Commons Attribution License, which permits unrestricted use, distribution, and reproduction in any medium, provided the original work is properly cited.

\begin{abstract}
The aim of this paper is to investigate whether urosepsis is related to irrigation pressure of ureteroscopy (URS) and evaluate the prognostic value of adrenomedullin (ADM) and atrial and brain natriuretic peptides (ANP and BNP) in URS-induced uroseptic patients. From July 2008 to October 2013, we enrolled 332 patients with untreated unilateral ureteral obstruction (UUO). The UUO group included three subgroups of, respectively, 118, 132, and 82 patients who underwent URS under intermittent stable irrigation pressure of, respectively, 80,120 , and $160 \mathrm{mmHg}$. The plasma concentrations of ADM, ANP, and BNP were measured in all subjects. URS was performed for all UUO patients; the values of the three peptides were measured again after URS. Irrigation pressure and stone size were independent risk factors of urosepsis. After URS, the plasma concentrations of ADM, ANP, and BNP were significantly higher in uroseptic patients. Moreover, the concentrations were significantly higher depending on the disease severity. Plasma concentrations of the three peptides were correlated with plasma ET concentration in the uroseptic patients. The areas under receiver operating characteristic (ROC) curve of ADM, ANP, and BNP for predicting urosepsis were $0.811,0.728$, and 0.764 , respectively. In conclusion, ADM, along with ANP and BNP, is valuable for prognosis in urosepsis secondary to URS which is associated with irrigation pressure.
\end{abstract}

\section{Introduction}

Urosepsis is defined as sepsis caused by infection of the urogenital tract and is a systemic response to infection [1]. Frequent causes for urosepsis are obstructive diseases of the urinary tract, such as ureteral stones, anomalies, stenosis, or tumor [2]. It can also occur after interventions in the urogenital tract, such as transrectal prostate biopsy, percutaneous nephrolithotomy (PCNL), or ureteroscopy (URS) [3-5]. Early diagnosis and timely intervention have great importance for urosepsis. Effective treatment in the early periods of urosepsis can prevent irreversible organ damage and reduce mortality. Therefore, finding useful biomarkers plays a crucial role in early diagnosis of urosepsis.

Adrenomedullin (ADM) was first isolated from human pheochromocytoma [6]. It has been detected in the plasma and other fluids of normal individuals [7]. Adrenomedullin possesses anti-inflammatory, bactericidal, positive inotropic, and, perhaps most importantly, vasodilatory activities [8].
Several clinical studies have demonstrated that ADM increases significantly in septic patients and is correlated with disease severity, which is valuable for prognosis in septic patients $[9,10]$.

Atrial and brain natriuretic peptides (ANP and BNP, resp.) are polypeptide hormones comprising the cardiacderived natriuretic peptide system which are involved in the long-term regulation of sodium and water balance, blood volume, and arterial pressure [11-13]. Increased plasma ANP and BNP have been identified as predictors of cardiac dysfunction in sepsis and prognosis in patients with congestive heart failure or ischemic heart disease $[14,15]$. Many reports indicate that ANP or BNP levels are elevated in septic patients and they can provide useful diagnostic and prognostic information in septic patients $[14,16,17]$.

To measure the plasma concentrations of ADM, ANP, and $\mathrm{BNP}$ and evaluate their prognostic value in uroseptic patients induced by URS, we measured the values of the three peptides in untreated patients with unilateral ureteral obstruction 
(UUO) secondary to ureteral stones and compared the results with those of healthy control subjects. Additionally, we measured the values of the three peptides after URS for UUO patients.

\section{Materials and Methods}

2.1. Study Subjects. This retrospective, case-control study was conducted in Renmin Hospital of Wuhan University between July 2008 and October 2013 in accordance with our institutional standards and under the appropriate license of the Ethics Committee of Renmin Hospital, as well as in adherence to national regulations. The study groups consisted of 90 healthy control subjects (50 men and 40 women, mean age $41.3 \pm 12.6$ years, range 18 to 66 years) and 332 patients with untreated UUO (185 men and 147 women, mean age $43.3 \pm 12.0$ years, range 18 to 68 years). In order to investigate whether urosepsis is related to irrigation pressure of ureteroscopy (URS), all UUO patients underwent URS with intermittent stable irrigation pressure of $80 \mathrm{mmHg}$ (group I, 66 men and 52 women, mean age $43.2 \pm 12.1$ years; range 18 to 68 years), $120 \mathrm{mmHg}$ (group II, 73 men and 59 women, mean age $43.3 \pm 11.6$ years; range 19 to 66 years), and $160 \mathrm{mmHg}$ (group III, 46 men and 36 women, mean age $43.6 \pm 12.8$ years; range 19 to 67 years), respectively. All patients agreed to participate in this study and provided written informed consent. The operation was performed by only one experienced surgeon. The irrigation pressure was randomly selected and the patients and surgeon were blind to it according to the double blind method. All patient information was anonymized and deidentified prior to analysis. Routine laboratory studies and image examinations were conducted before URS. The inclusion criteria for UUO subjects were as follows: simple unilateral ureteral stone, without urinary tract infections by urinalysis (the presence of 10 urine leukocytes/HPF and no microorganisms), without any symptoms of urogenital tract infections, without antimicrobial prophylaxis before the procedure. Ureteroscopy was performed using a Wolf rigid ureteroscope. UUO was definitely diagnosed on the basis of intravenous pyelonephrography or computed tomography. All patients undergoing laser lithotripsy had a double-J stent and a Foley catheter placed at the end of the procedure. The indwelling Foley catheter was drawn within 72 hours. All uroseptic patients were symptomatic or signs of potential sepsis were present within 24 hours after URS. They fulfilled the systemic inflammatory response syndrome (SIRS) criteria defined by the American College of Chest Physicians/Society of Critical Care Medicine [18], regardless of the procedure duration and residual stones. Patients who developed sepsis were treated with vasopressors such as phenylephrine and norepinephrine to sustain blood pressure when necessary. None of these UUO patients had clinical evidence of active infection, malignant cancer of any type, acquired immunodeficiency syndrome (AIDS), end-stage renal or liver disease, diabetes, pulmonary disease, valvular heart disease, congenital heart disease, acute myocarditis, angina pectoris, myocardial infarction, essential hypertension, or other diseases. Control subjects were age- and gender-matched healthy subjects who had been hospitalized for a health checkup.

2.2. Assay Procedures. Venous blood samples were drawn from an antecubital vein within 24 hours for nonseptic subjects or septic patients before vasopressor treatment and were transferred to ice-chilled tubes containing Trasylol $(500 \mathrm{KIU} / \mathrm{mL})$ and ethylenediaminetetraacetic acid (EDTA, $1 \mathrm{~g} / \mathrm{L})$. They were then centrifuged at 3,000 rpm for $15 \mathrm{~min}$ at $4^{\circ} \mathrm{C}$ and the plasma was immediately frozen and stored in polypropylene tubes at $-80^{\circ} \mathrm{C}$ until radioimmunoassay (RIA).

Baseline clinical data were recorded as follows: endotoxin (ET) using the Pyrochrome test kit (Pyroquant Diagnostik GmbH, Mörfelden, Germany), white blood cell counts (WBC) using a Sysmex SE-9000 analyzer (Toa Medical Instruments, Kobe, Japan), C-reactive protein (CRP) using an immunoturbidimetric assay (Modular Analytics P, Roche Diagnostics, Mannheim, Germany), lactate (LAC) using enzymatic method (Modular Analytics P, Roche Diagnostics, Mannheim, Germany), and procalcitonin (PCT) using an immunoassay analyzer (Block Scientific, Bohemia, NY).

The plasma ADM levels were measured with specific RIA for human ADM (ADM RIA SHIONOGI, Shionogi Pharmaceutical, Co., Ltd., Osaka, Japan). The intra- and interassay coefficients of variation were $3.8 \%$ to $7.9 \%$ and $4.5 \%$ to $8.8 \%$, respectively. All assays were performed in duplicate. ADM concentrations were expressed as ng/L.

The plasma ANP concentrations were measured with a specific immunoradiometric assay for human ANP (Shiono RIA ANP kit, Shionogi and Co., Osaka, Japan). The intraand interassay coefficients of variation were $4.7 \%$ to $9.8 \%$ and $5.9 \%$ to $11.6 \%$, respectively. The plasma BNP concentrations were measured by a method similar to that for ANP, developed by the same company (Shiono RIA BNP kit). The intraand interassay coefficients of variation were $5.8 \%$ to $10.7 \%$ and $6.5 \%$ to $12.5 \%$, respectively. All assays were performed in duplicate. The concentrations of ANP and BNP were expressed as $\mathrm{ng} / \mathrm{L}$.

2.3. Statistical Analysis. All continuous data were expressed as mean \pm SD and analyzed with SPSS software, version 19.0 (SPSS Inc., Chicago, IL). Comparisons between two variables were performed with unpaired $t$-test or Mann-Whitney $U$ test. Multiple comparisons were evaluated with analysis of variance followed by Student-Newman-Keuls or KruskalWallis method. The significance of differences between paired variables was determined by paired $t$-test or Wilcoxon test. Categorical variables were assessed by the chi-square test or Fisher's exact test. Stepwise multiple linear regression analysis was used to evaluate the most important factor for ADM, ANP, and BNP. The correlation between two variables was done by linear regression analysis and further confirmed by Spearman's rank test. Receiver operating characteristic (ROC) curves were used to predict urosepsis and determine the cutoff values. Kaplan-Meier curves were plotted according to the identified cutoff values of ADM, ANP, and BNP and further confirmed by log-rank test. A 2 -sided $p$ value less than 0.05 was considered to indicate statistical significance. 
TABLE 1: Baseline characteristics of study subjects.

\begin{tabular}{|c|c|c|c|c|c|}
\hline \multirow{2}{*}{ Characteristics } & \multirow{2}{*}{ Control $(n=90)$} & \multicolumn{3}{|c|}{ UUO $(n=332)$} & \multirow{2}{*}{$p$ value } \\
\hline & & Group I $(n=118)$ & Group II $(n=132)$ & Group III $(n=82)$ & \\
\hline Age (years) & $41.3 \pm 12.6$ & $43.2 \pm 12.1$ & $43.3 \pm 11.6$ & $43.6 \pm 12.8$ & $>0.05$ \\
\hline \multicolumn{6}{|l|}{ Gender } \\
\hline Men & $50(55.6 \%)$ & $66(55.9 \%)$ & $73(55.3 \%)$ & $46(56.1 \%)$ & \multirow{2}{*}{$>0.05$} \\
\hline Women & $40(44.4 \%)$ & $52(44.1 \%)$ & $59(44.7 \%)$ & $36(43.9 \%)$ & \\
\hline \multicolumn{6}{|l|}{ Side } \\
\hline Left & - & $60(50.8 \%)$ & $67(50.8 \%)$ & $42(51.2 \%)$ & \multirow{2}{*}{$>0.05$} \\
\hline Right & - & $58(49.2 \%)$ & $65(49.2 \%)$ & $40(48.8 \%)$ & \\
\hline \multicolumn{6}{|l|}{ Stone site } \\
\hline Proximal ureter & - & $37(31.4 \%)$ & $40(30.3 \%)$ & $25(30.5 \%)$ & \multirow{3}{*}{$>0.05$} \\
\hline Mid ureter & - & $41(34.7 \%)$ & $45(34.1 \%)$ & $28(34.1 \%)$ & \\
\hline Distal ureter & & $40(33.9 \%)$ & $47(35.6 \%)$ & $29(35.4 \%)$ & \\
\hline Stone size (mm) & - & $9.2 \pm 4.1$ & $9.6 \pm 4.5$ & $10.3 \pm 5.2$ & $>0.05$ \\
\hline WBC $\left(\times 10^{9} / \mathrm{L}\right)$ & $7.1 \pm 1.5$ & $7.2 \pm 1.6$ & $6.8 \pm 1.5$ & $7.0 \pm 1.7$ & $>0.05$ \\
\hline ET (ng/L) & $3.6 \pm 1.4$ & $3.5 \pm 1.2$ & $3.6 \pm 1.6$ & $3.5 \pm 1.7$ & $>0.05$ \\
\hline CRP (mg/L) & $6.0 \pm 2.2$ & $5.8 \pm 2.1$ & $5.9 \pm 2.1$ & $6.2 \pm 2.1$ & $>0.05$ \\
\hline $\mathrm{LAC}(\mathrm{mmol} / \mathrm{L})$ & $1.1 \pm 0.5$ & $1.2 \pm 0.6$ & $1.3 \pm 0.6$ & $1.2 \pm 0.5$ & $>0.05$ \\
\hline PCT (ng/mL) & $0.24 \pm 0.13$ & $0.23 \pm 0.12$ & $0.25 \pm 0.13$ & $0.22 \pm 0.10$ & $>0.05$ \\
\hline $\operatorname{Scr}(\mu \mathrm{mol} / \mathrm{L})$ & $75 \pm 12$ & $77 \pm 14$ & $76 \pm 13$ & $74 \pm 11$ & $>0.05$ \\
\hline $\operatorname{Ccr}(\mathrm{mL} / \mathrm{min})$ & $100 \pm 10$ & $101 \pm 12$ & $99 \pm 11$ & $102 \pm 14$ & $>0.05$ \\
\hline
\end{tabular}

UUO: unilateral ureteral obstruction; WBC: white blood cell count; ET: endotoxin; CRP: C-reactive protein; LAC: lactate; PCT: procalcitonin; Scr: serum creatinine; Ccr: creatinine clearance.

The normal values are as follows: WBC, 4.0 10.0 × 10 $/ \mathrm{L} ; \mathrm{ET}, 0 \sim 10 \mathrm{ng} / \mathrm{L} ; \mathrm{CRP}, 0 \sim 10 \mathrm{mg} / \mathrm{L} ; \mathrm{LAC}, 0 \sim 2.4 \mathrm{mmol} / \mathrm{L} ; \mathrm{PCT}, 0 \sim 0.5 \mathrm{ng} / \mathrm{mL} ; \mathrm{Scr}, 53 \sim 106 \mu \mathrm{mol} / \mathrm{L}$ (male), 44 97 $\mu \mathrm{mol} / \mathrm{L}$ (female); Ccr, 80 120 mL/min.

\section{Results}

Table 1 shows the baseline characteristics of the study groups. There were no significant differences in age, sex distribution, WBC, and plasma concentrations of ET, CRP, LAC, PCT, Scr, and $\mathrm{Ccr}$ among the four groups. No significant differences were observed in stone side distribution, stone site, and stone size among the three UUO subgroups.

After URS, the uroseptic rates of the three UUO subgroups were $8.5 \%(10 / 118), 18.2 \%$ (24/132), and 30.5\% (25/82), respectively, which were significantly higher and higher in proportion to the irrigation pressure $(p<0.05)$. However, there were no significant differences in age, sex distribution, Scr, and Ccr among the three uroseptic groups (data not shown).

We analyzed the risk factors of urosepsis by stepwise multiple logistic regression analysis which revealed that stone size $(B=0.695, \mathrm{OR}=2.004, p=0.024)$ and irrigation pressure $(B=0.750, \mathrm{OR}=2.118, p=0.000)$ were the most important independent factors of urosepsis, when age, sex, stone side, stone site, stone size, and irrigation pressure were taken into account.

Table 2 shows the clinical parameters at diagnosis and after URS in uroseptic patients. As expected, WBC and plasma concentrations of ET, CRP, LAC, and PCT were significantly higher after URS than at diagnosis $(p<0.05)$. However, Scr and Ccr remained unchanged after URS.

The plasma concentrations of ADM, ANP, and BNP in controls and uroseptic patients before and after URS are depicted in Figures 1(a)-1(c). The mean value of ADM was significantly higher in uroseptic group I after URS (50.19 \pm $20.67 \mathrm{ng} / \mathrm{L})$ than before URS (19.08 $\pm 7.36 \mathrm{ng} / \mathrm{L})$ and in controls $(18.50 \pm 6.46 \mathrm{ng} / \mathrm{L})(p<0.05)$. There was no significant difference in mean ADM value between uroseptic group I before URS and controls. The mean values of ANP in controls and uroseptic group I before and after URS were $23.63 \pm 8.98,22.56 \pm 8.70$, and $82.91 \pm 30.43 \mathrm{ng} / \mathrm{L}$, respectively, while the mean values of BNP were $12.72 \pm 5.52,13.24 \pm 4.11$, and $137.97 \pm 57.79 \mathrm{ng} / \mathrm{L}$, respectively. Similar changes were observed in mean values of ANP and BNP in uroseptic group I. Similar changes were found in mean values of the three peptides in uroseptic groups II and III.

Table 3 shows the clinical parameters of uroseptic patients depending on the disease severity. There were no significant differences in age, sex distribution, WBC, Scr, and Ccr among the three groups. No significant differences were detected in plasma concentrations of CRP, LAC, and PCT between sepsis and severe sepsis, while a significant difference was observed in plasma ET $(p<0.05)$. However, plasma concentrations of ET, CRP, LAC, and PCT were significantly higher in septic shock than in sepsis $(p<0.05)$.

As shown in Table 4, ET was the most important factor associated with ADM, ANP, and BNP in the uroseptic patients. Stepwise multiple regression analysis of independent parameters (WBC, ET, CRP, LAC, and PCT) related to the values of plasma ADM, ANP, and BNP was also conducted. 
TABLE 2: Parameters of uroseptic patients before and after URS.

\begin{tabular}{lcccccc}
\hline \multirow{2}{*}{ Parameters } & \multicolumn{2}{c}{ Uroseptic group I $(n=10)$} & \multicolumn{2}{c}{ Uroseptic group II $(n=24)$} & \multicolumn{2}{c}{ Uroseptic group III $(n=25)$} \\
& At diagnosis & After URS & At diagnosis & After URS & At diagnosis & After URS \\
\hline WBC $\left(\times 10^{9} / \mathrm{L}\right)$ & $7.5 \pm 1.8$ & $13.4 \pm 5.8^{*}$ & $6.9 \pm 1.6$ & $15.6 \pm 6.6^{*}$ & $7.2 \pm 1.9$ & $17.2 \pm 9.4^{*}$ \\
ET $(\mathrm{ng} / \mathrm{L})$ & $3.8 \pm 1.9$ & $22.1 \pm 10.2^{*}$ & $3.4 \pm 1.8$ & $29.5 \pm 14.4^{*}$ & $3.7 \pm 1.7$ & $36.4 \pm 15.6^{*}$ \\
CRP $(\mathrm{mg} / \mathrm{L})$ & $5.0 \pm 2.4$ & $59.2 \pm 19.0^{*}$ & $5.2 \pm 2.0$ & $109.7 \pm 48.9^{*}$ & $6.0 \pm 2.2$ & $145.9 \pm 59.3^{*}$ \\
LAC $(\mathrm{mmol} / \mathrm{L})$ & $1.4 \pm 0.6$ & $2.4 \pm 0.9^{*}$ & $1.1 \pm 0.6$ & $2.3 \pm 0.8^{*}$ & $1.1 \pm 0.5$ & $2.5 \pm 0.9^{*}$ \\
PCT $(\mathrm{ng} / \mathrm{mL})$ & $0.20 \pm 0.13$ & $0.59 \pm 0.23^{*}$ & $0.25 \pm 0.14$ & $0.58 \pm 0.24^{*}$ & $0.24 \pm 0.11$ & $0.60 \pm 0.24^{*}$ \\
Scr $(\mu \mathrm{mol} / \mathrm{L})$ & $73 \pm 8$ & $74 \pm 10$ & $75 \pm 10$ & $77 \pm 13$ & $74 \pm 9$ & $75 \pm 12$ \\
Ccr $(\mathrm{mL} / \mathrm{min})$ & $101 \pm 8$ & $100 \pm 11$ & $100 \pm 9$ & $99 \pm 12$ & $99 \pm 10$ & $97 \pm 14$ \\
\hline
\end{tabular}

URS: ureteroscopy; WBC: white blood cell count; ET: endotoxin; CRP: C-reactive protein; LAC: lactate; PCT: procalcitonin; Scr: serum creatinine; Ccr: creatinine clearance.

${ }^{*} p<0.05$, compared with subjects at diagnosis.

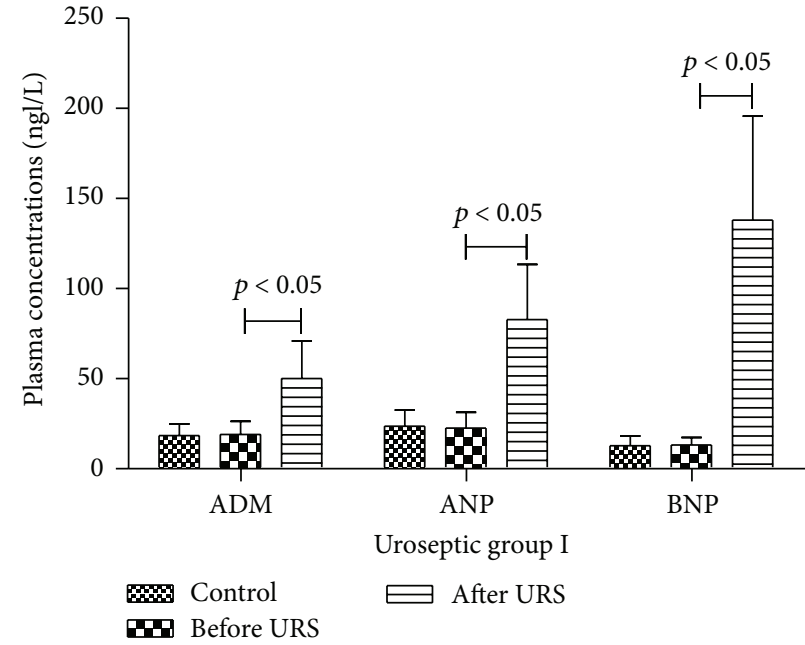

(a)

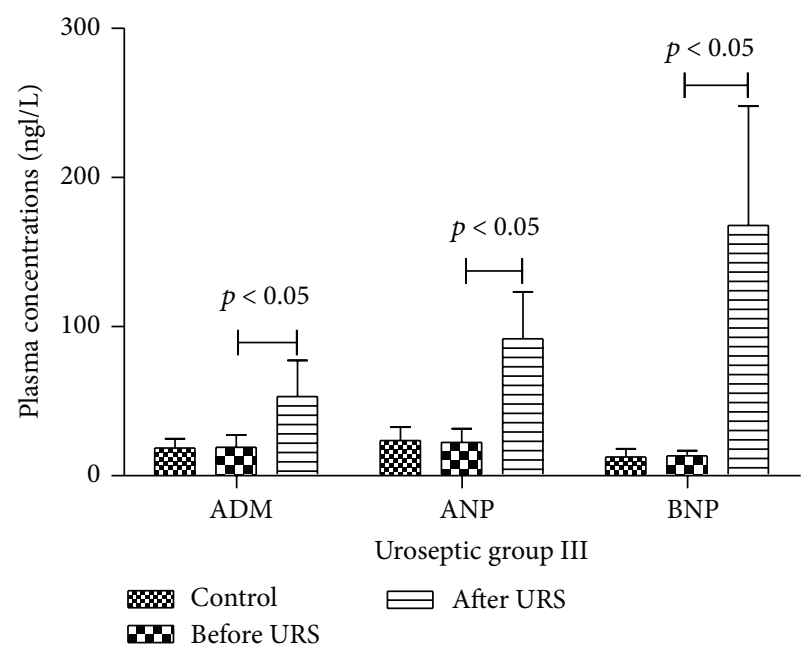

(c)

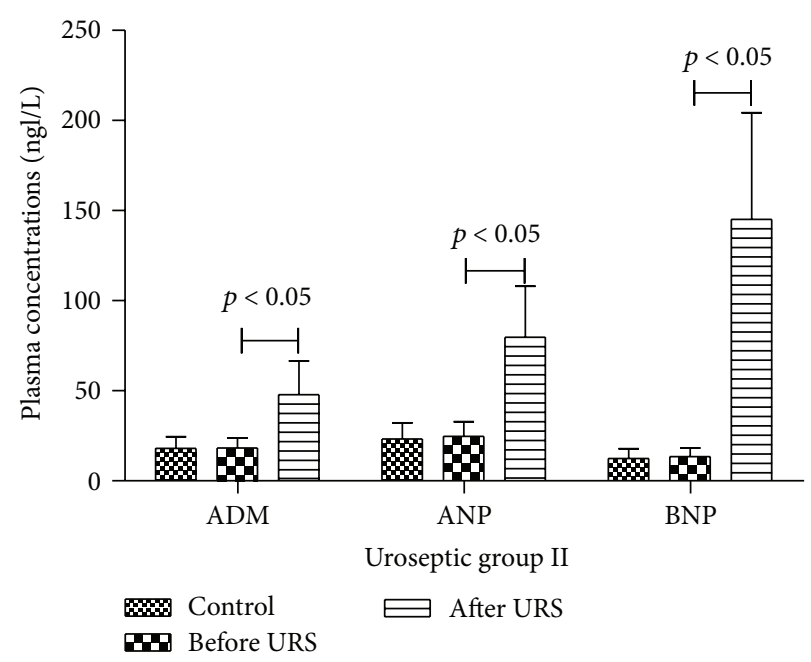

(b)

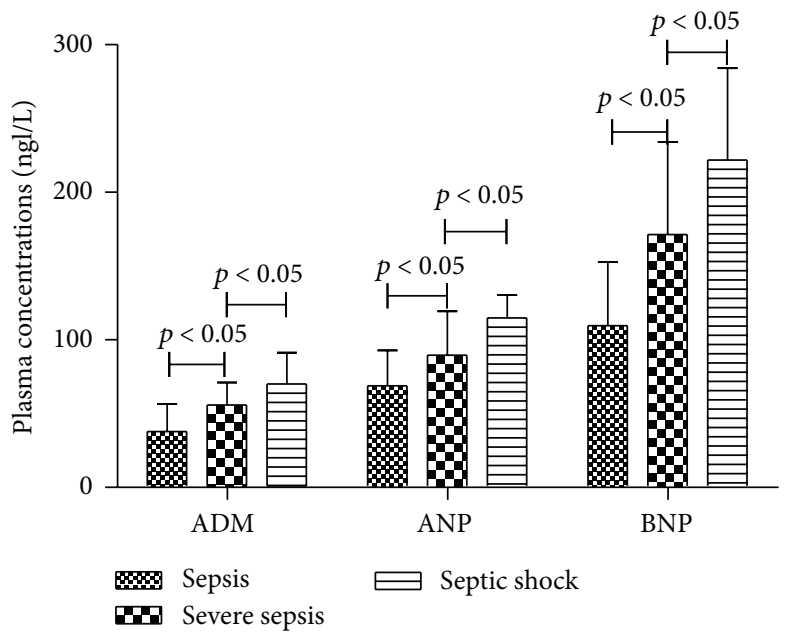

(d)

Figure 1: (a) Plasma concentrations of ADM, ANP, and BNP in controls and uroseptic group I before and after URS under $80 \mathrm{mmHg}$. (b) Plasma concentrations of ADM, ANP, and BNP in controls and uroseptic group II before and after URS under $120 \mathrm{mmHg}$. (c) Plasma concentrations of ADM, ANP, and BNP in controls and uroseptic group III before and after URS under $160 \mathrm{mmHg}$. (d) Plasma concentrations of ADM, ANP, and BNP in URS-induced sepsis, severe sepsis, and septic shock. 


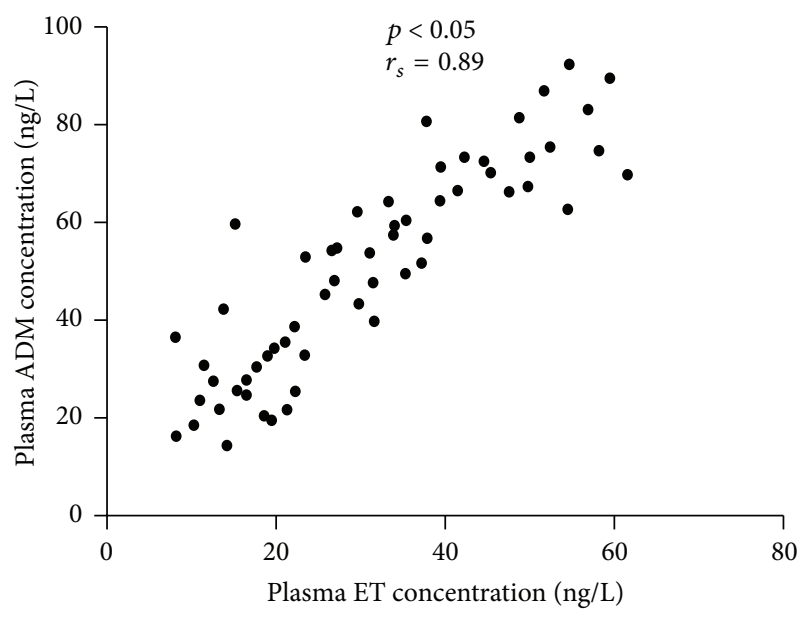

(a)

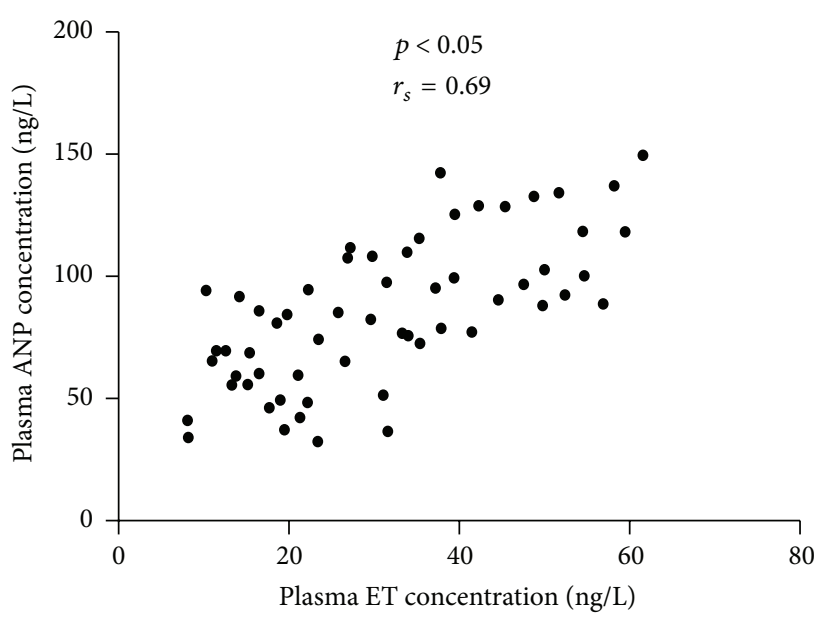

(b)

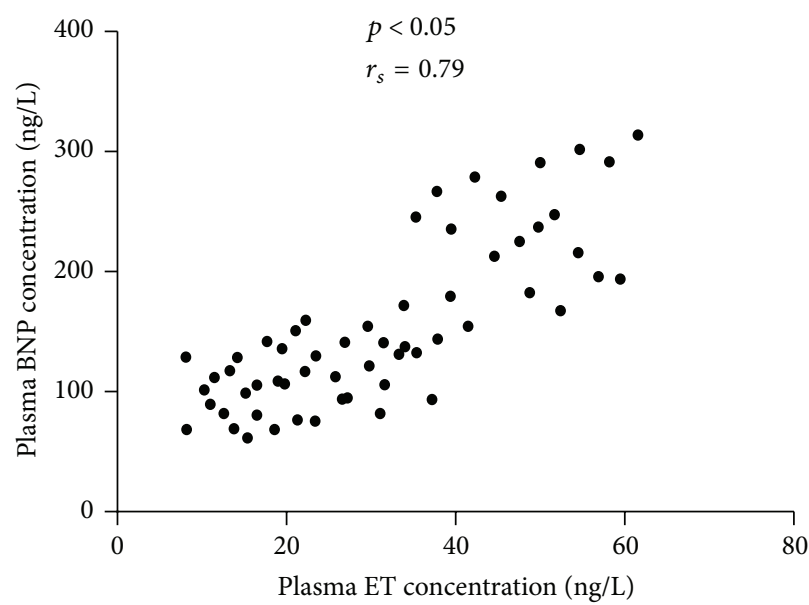

(c)

FIGURE 2: Relationship of plasma ET concentration to values of ADM (a), ANP (b), and BNP (c) in all uroseptic patients.

TABLE 3: Parameters of uroseptic patients depending on the disease severity after URS.

\begin{tabular}{lccc}
\hline Parameters & $\begin{array}{c}\text { Sepsis } \\
(n=25)\end{array}$ & $\begin{array}{c}\text { Severe sepsis } \\
(n=24)\end{array}$ & $\begin{array}{c}\text { Septic shock } \\
(n=10)\end{array}$ \\
\hline Age (years) & $44.2 \pm 12.9$ & $45.8 \pm 12.2$ & $45.1 \pm 13.5$ \\
Gender $($ male $:$ female $)$ & $12: 13$ & $13: 11$ & $4: 6$ \\
WBC $\left(\times 10^{9} / \mathrm{L}\right)$ & $13.7 \pm 9.6$ & $17.6 \pm 6.1$ & $17.5 \pm 5.4$ \\
ET $(\mathrm{ng} / \mathrm{L})$ & $21.9 \pm 10.0$ & $35.3 \pm 15.1^{*}$ & $44.4 \pm 11.4^{*}$ \\
CRP $(\mathrm{mg} / \mathrm{L})$ & $89.5 \pm 46.8$ & $116.8 \pm 50.9$ & $183.1 \pm 51.1^{* \#}$ \\
LAC $(\mathrm{mmol} / \mathrm{L})$ & $2.1 \pm 0.7$ & $2.6 \pm 0.8$ & $2.9 \pm 0.8^{*}$ \\
PCT $(\mathrm{ng} / \mathrm{mL})$ & $0.50 \pm 0.23$ & $0.62 \pm 0.22$ & $0.74 \pm 0.19^{*}$ \\
Scr $(\mu \mathrm{mol} / \mathrm{L})$ & $73 \pm 11$ & $76 \pm 13$ & $80 \pm 15$ \\
Ccr $(\mathrm{mL} / \mathrm{min})$ & $101 \pm 8$ & $98 \pm 11$ & $95 \pm 14$ \\
\hline URS $(\mathrm{wr}$ &
\end{tabular}

URS: ureteroscopy; WBC: white blood cell count; ET: endotoxin; CRP: Creactive protein; LAC: lactate; PCT: procalcitonin; Scr: serum creatinine; Ccr: creatinine clearance.

${ }^{*} p<0.05$, compared with sepsis; ${ }^{*} p<0.05$, compared with severe sepsis.
Figure 1(d) shows the plasma concentrations of ADM, ANP, and BNP in uroseptic patients depending on the disease severity. The mean value of ADM was significantly higher in septic shock $(70.05 \pm 21.21 \mathrm{ng} / \mathrm{L})$ than in severe sepsis $(55.90 \pm 15.31 \mathrm{ng} / \mathrm{L})$ and sepsis $(37.75 \pm 18.84 \mathrm{ng} / \mathrm{L})(p<0.05)$. There was also a significant difference in mean ADM value between severe sepsis and sepsis $(p<0.05)$. Similar changes were found in mean values of ANP and BNP.

Scatterplots of Figure 2 show relationship of plasma ET concentration to plasma concentrations of ADM (a), ANP (b), and BNP (c) in uroseptic patients. Plasma ET concentration was positively related to plasma concentrations of $\mathrm{ADM}$, ANP, and BNP.

The ROC curves of WBC, ET, CRP, LAC, PCT, ADM, ANP, and BNP for urosepsis are shown in Figure 3. The AUCs are listed in Table 5. The AUC of ADM was 0.811 which was higher than those of WBC (0.712), ET (0.719), CRP (0.758), LAC (0.787), PCT (0.793), ANP (0.728), and BNP (0.764). 

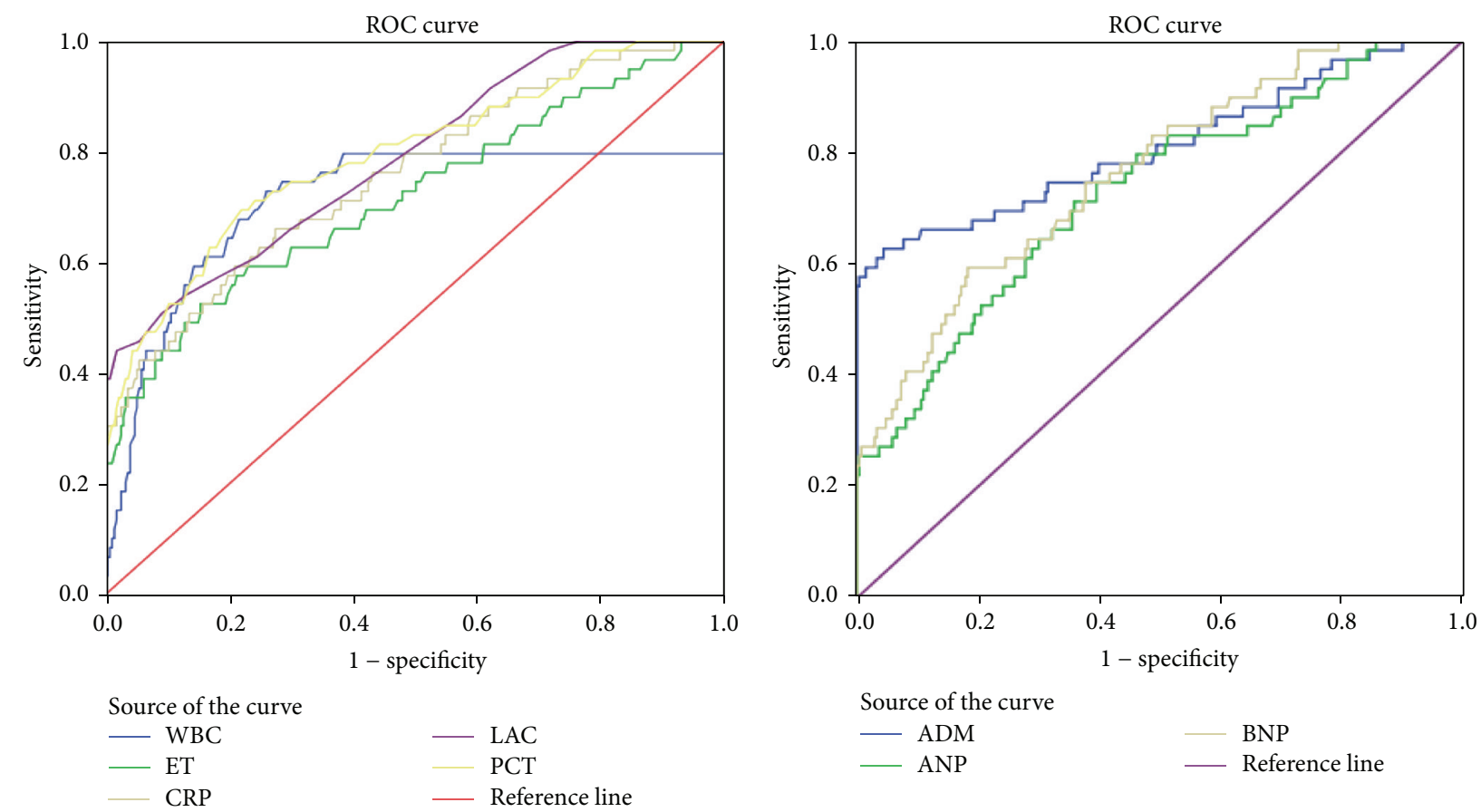

Figure 3: The ROC curves of ET, WBC, CRP, LAC, PCT, ADM, ANP, and BNP for predicting URS-induced urosepsis in UUO patients.

TABLE 4: Stepwise multiple regression analysis of significant factors for ADM, ANP, and BNP in uroseptic patients.

\begin{tabular}{|c|c|c|c|c|c|c|c|c|c|}
\hline \multirow{2}{*}{ Variables } & \multicolumn{3}{|c|}{$\mathrm{ADM}(\mathrm{ng} / \mathrm{L})$} & \multicolumn{3}{|c|}{ ANP (ng/L) } & \multicolumn{3}{|c|}{ BNP (ng/L) } \\
\hline & $B$ & $t$ & $p$ & $B$ & $t$ & $p$ & $B$ & $t$ & $p$ \\
\hline $\mathrm{WBC}\left(\times 10^{9} / \mathrm{L}\right)$ & 0.020 & 0.106 & 0.916 & -0.297 & -0.694 & 0.491 & -0.567 & -0.689 & 0.494 \\
\hline ET (ng/L) & 0.847 & 4.835 & 0.000 & 0.979 & 2.445 & 0.018 & 3.797 & 4.947 & 0.000 \\
\hline CRP (mg/L) & 0.034 & 1.058 & 0.295 & 0.100 & 1.367 & 0.177 & -0.120 & -0.858 & 0.395 \\
\hline $\mathrm{LAC}(\mathrm{mmol} / \mathrm{L})$ & 4.404 & 1.964 & 0.055 & 7.101 & 1.386 & 0.172 & 12.994 & 1.323 & 0.192 \\
\hline PCT (ng/mL) & 13.557 & 1.550 & 0.127 & -7.611 & -0.381 & 0.705 & -16.361 & -0.427 & 0.671 \\
\hline
\end{tabular}

ADM: adrenomedullin; ANP: atrial natriuretic peptide; BNP: brain natriuretic peptide; WBC: white blood cell count; ET: endotoxin; CRP: C-reactive protein; LAC: lactate; PCT: procalcitonin.

TABLE 5: The AUCs of WBC, ET, CRP, LAC, PCT, ADM, ANP, and BNP.

\begin{tabular}{lccccc}
\hline \multirow{2}{*}{ Variables } & \multirow{2}{*}{ Area } & SE & $p$ & \multicolumn{2}{c}{$95 \%$ CI } \\
& & & & Lower & Upper \\
\hline WBC $\left(\times 10^{9} / \mathrm{L}\right)$ & 0.712 & 0.049 & 0.000 & 0.615 & 0.809 \\
ET $(\mathrm{ng} / \mathrm{L})$ & 0.719 & 0.041 & 0.000 & 0.639 & 0.799 \\
CRP $(\mathrm{mg} / \mathrm{L})$ & 0.758 & 0.037 & 0.000 & 0.686 & 0.830 \\
LAC $(\mathrm{mmol} / \mathrm{L})$ & 0.787 & 0.034 & 0.000 & 0.720 & 0.854 \\
PCT $(\mathrm{ng} / \mathrm{mL})$ & 0.793 & 0.035 & 0.000 & 0.724 & 0.862 \\
ADM $(\mathrm{ng} / \mathrm{L})$ & 0.811 & 0.038 & 0.000 & 0.737 & 0.885 \\
ANP $(\mathrm{ng} / \mathrm{L})$ & 0.728 & 0.037 & 0.000 & 0.655 & 0.801 \\
BNP $(\mathrm{ng} / \mathrm{L})$ & 0.764 & 0.034 & 0.000 & 0.697 & 0.831 \\
\hline
\end{tabular}

WBC: white blood cell count; ET: endotoxin; CRP: C-reactive protein; LAC: lactate; PCT: procalcitonin; ADM: adrenomedullin; ANP: atrial natriuretic peptide; BNP: brain natriuretic peptide.
Kaplan-Meier curves for ADM, ANP, and BNP are depicted in Figure 4 . The cutoff values of ADM, ANP, and BNP were $41.925,68.565$, and $128.575 \mathrm{ng} / \mathrm{L}$, respectively, for prognosis in uroseptic patients. The survival rates were $64.9 \%, 66.7 \%$, and $62.9 \%$, respectively, whose values of the three peptides were above the cutoff values, whereas the survival rates were $90.9 \%, 94.1 \%$, and $91.7 \%$, respectively, whose values of the three peptides were below the cutoff values. There was a significant difference in survival rates between the groups above and below the cutoff values $(p<$ 0.05).

\section{Discussion}

Ureteral calculi represent a common condition that urologists encounter in everyday practice. Ureteroscopy is one of the 

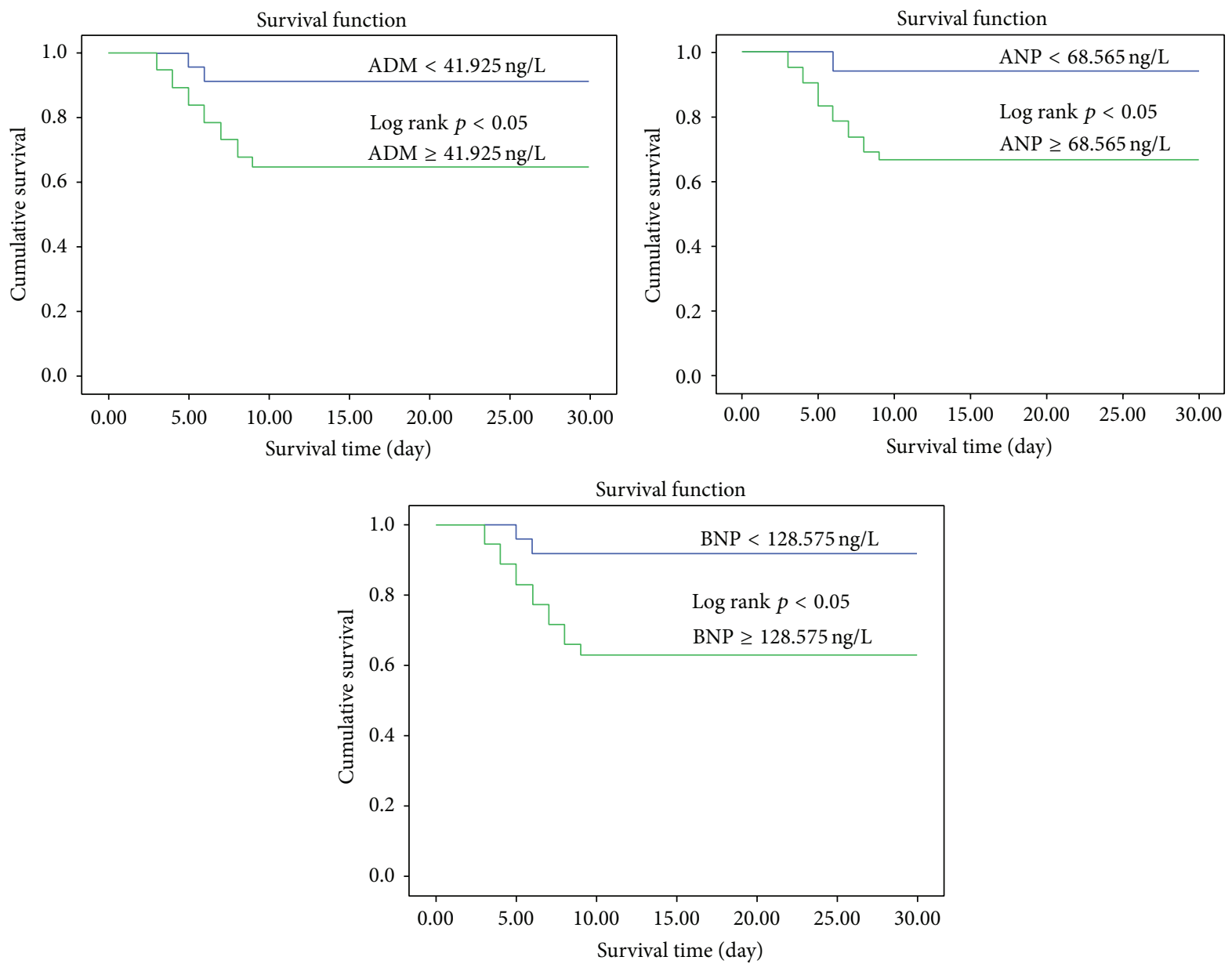

FIGURE 4: Kaplan-Meier curves for 69 uroseptic patients subdivided into two groups according to the cutoff values of ADM, ANP, and BNP in plasma.

most important treatment options for ureteral calculi that do not pass spontaneously or are unlikely to do so [19]. This procedure carries the risk of postoperative urosepsis affecting UUO patients undergoing ureteroscopy and laser lithotripsy [20]. Urosepsis, uroseptic shock, and the ensuing multiple organ failure continue to be the most common causes of death in critically ill patients of urological department admitted to intensive care unit (ICU) [21]. In urosepsis, as in other types of sepsis, the severity of sepsis depends mostly upon the host response [22]. Human ADM, consisting of 52 amino acids, has a ring structure formed by a disulfide bond and an amidated carboxyl terminus and belongs to a family of calcitonin gene-related peptides [23]. Nowadays, it has been demonstrated that ADM can be synthesized by various other tissues including endothelial and vascular smooth muscle cells, myocardium, and central nervous system [24]. It has multiple functions in a wide range of tissues and acts mainly as a vasodilatory and proliferation-inhibitory factor in cardiovascular system [25]. It was recently reported that ADM plays a central role in initiating the hyperdynamic response during the early stages of sepsis and was a useful predictor for development of severe sepsis and septic shock [10,26]. The ANP and BNP are similar to ADM in cardiovascular effects including natriuresis, diuresis, and vasodilatation, thereby reducing fluid volume and blood pressure [13, 27]. ANP is a 28-amino-acid peptide chiefly synthesized and released by atrial myocytes in response to atrial distension and stretch, whereas BNP is a 32-amino-acid peptide synthesized and released by ventricular myocytes in response to ventricular stretch or pressure overload [28]. Pro-ANP is a valuable biomarker for prediction of severity of septic patients $[29,30]$. The plasma BNP concentrations were increased in patients with severe sepsis or septic shock and poor outcome was associated with high BNP levels [31, 32].

In the current study, the uroseptic rates were significantly higher and higher in proportion to the irrigation pressure. This result was further confirmed by logistic regression analysis of risk factors for urosepsis which showed irrigation pressure was an independent risk factor. Therefore, this result may provide a guide that it is necessary to perform URS under lower irrigation pressure in the clinical practice. Moreover, stone size was another independent risk factor for urosepsis. It can be easily explained that the operation time is longer with bigger stone size.

In our study, plasma levels of ADM, ANP, and BNP were higher in uroseptic patients after URS than before URS 
and in controls, as well as WBC and plasma concentrations of ET, CRP, LAC, and PCT, but Scr and Ccr remained unchanged. No significant differences were found between uroseptic patients before URS and controls. Moreover, the plasma concentrations of ADM, ANP, and BNP were related to the severity of disease, as well as plasma concentrations of ET, CRP, LAC, and PCT $[9,33,34]$. It can be inferred that WBC and plasma concentrations of ET, CRP, LAC, and PCT had some predictive value for urosepsis and disease severity, in agreement with the literature [35]. ADM, along with ANP and BNP, may participate in initiating the hyperdynamic response during the early stages of sepsis because of their similar physiological functions, in agreement with the literature $[16,26]$. Endotoxin was identified as the most important factor in uroseptic patients. This result was confirmed by stepwise multiple regression analysis of independent parameters related to plasma concentrations of ADM, ANP, and BNP. Plasma endotoxin concentration was not only correlated with values of ADM but also related to the values of ANP and BNP in uroseptic patients. We can infer that URS may cause endotoxin absorption which is proportional to the irrigation pressure. The elevated plasma endotoxin concentration may subsequently result in ADM secretion and myocardial cell injury which are responsible for the elevated plasma levels of ADM, ANP, and BNP. Indeed, the mechanism of ADM secretion in large part relates to the effects of lipopolysaccharide (LPS) stimulation which is the most important ingredient of endotoxin. Moreover, it can be inferred that urosepsis secondary to endotoxin absorption can lead to endotoxic cardiomyopathy.

Biomarkers play important roles in diagnosis, differential diagnosis, risk stratification, therapeutic monitoring, and prognosis in sepsis. Many features of pathophysiologic progression correlate with the severity and outcome of the disease and become candidate prognostic biomarkers [36]. Our study showed that the older biomarkers of WBC and plasma concentrations of ET, CRP, LAC, and PCT were predictive indicators of urosepsis according to their AUCs. Our data seem to be compatible with the previous report [35]. The predictive value of PCT is still superior to the other biomarkers. However, the most valuable predictor is $\mathrm{ADM}$ and ANP and BNP also have some predictive value in urosepsis according to their AUCs, in agreement with the literature $[10,30,32]$.

Several clinical studies have demonstrated that ADM, ANP, and BNP are predictors of adverse outcome in patients with sepsis, but most of these studies were conducted in the ICU and contained relatively small sample sizes. The prognostic value of ADM, ANP, and BNP in uroseptic patients induced by URS in the urological department is still undefined.

Our results suggest that the prognostic value of $\mathrm{ADM}$ is superior to ANP and BNP, and ADM, ANP, and BNP are robust independent predictors of in-hospital death in uroseptic patients. In uroseptic patients with ADM, ANP, and BNP levels above the cutoff values of our study, the in-hospital mortality was $35.1 \%, 33.3 \%$, and $36.1 \%$, respectively, with all patients dying within the first 10 days. In uroseptic patients with ADM, ANP, and BNP levels below the cutoff values, survival rates were $90.9 \%, 94.1 \%$, and $91.7 \%$, respectively, at the 30-day follow-up. These results demonstrate that ADM, ANP, and BNP are strong predictors of adverse outcome in patients with urosepsis.

\section{Conclusions}

In summary, with the physiological roles of ADM taken together, our study shows that ADM, along with ANP and BNP, may participate in initiating the hyperdynamic response during the early stages of sepsis in uroseptic patients. In addition, ADM, ANP, and BNP are strong predictors of adverse outcome in patients with urosepsis.

\section{Ethical Approval}

This study has been approved by the Ethics Committee of Renmin Hospital of Wuhan University, in adherence to China Association for Ethical Studies.

\section{Consent}

All subjects agreed to participate in this study and provided written informed consent which was kept on file after the ethics committee approved this consent procedure.

\section{Conflict of Interests}

The authors report no conflict of interests regarding the publication of this paper.

\section{Authors' Contribution}

Wei Hu was responsible for project development, data collection, data analysis, and paper writing. Pang-hu Zhou was in charge of project development and data collection. Xiao-bin Zhang was responsible for project development. Wei Wang and Lijun Zhang carried out data collection. Wei $\mathrm{Hu}$ and Pang-hu Zhou contributed equally to this study.

\section{Acknowledgments}

Thanks are due to the Department of Urology in Renmin Hospital of Wuhan University. This study was supported by grants from the National Science Fund Project of China (no. 81501921) and the Doctor Research Fund Project of Wuhan University of China (no. 2012302020203).

\section{References}

[1] F. M. Wagenlehner, C. Lichtenstern, C. Rolfes et al., "Diagnosis and management for urosepsis," International Journal of Urology, vol. 20, no. 10, pp. 963-970, 2013.

[2] F. M. E. Wagenlehner, C. Lichtenstern, M. A. Weigand, and W. Weidner, "Urosepsis and treatment," Der Urologe, vol. 49, no. 5, pp. 618-622, 2010. 
[3] F. M. E. Wagenlehner, A. Pilatz, P. Waliszewski, T. Dansranjavin, and W. Weidner, "Infection and sepsis prevention in prostate biopsy," Der Urologe, vol. 52, no. 10, pp. 1459-1468, 2013.

[4] S. Kumar, S. Bag, R. Ganesamoni, A. K. Mandal, N. Taneja, and S. K. Singh, "Risk factors for urosepsis following percutaneous nephrolithotomy: role of 1 week of nitrofurantoin in reducing the risk of urosepsis," Urological Research, vol. 40, no. 1, pp. 7986, 2012.

[5] J. M. Watson, C. Chang, J. G. Pattaras, and K. Ogan, "Same session bilateral ureteroscopy is safe and efficacious," The Journal of Urology, vol. 185, no. 1, pp. 170-174, 2011.

[6] K. Kitamura, K. Kangawa, M. Kawamoto et al., "Adrenomedullin: a novel hypotensive peptide isolated from human pheochromocytoma. 1993," Biochemical and Biophysical Research Communications, vol. 425, no. 3, pp. 548-555, 2012.

[7] H. K. Wong, T. T. Cheung, and B. M. Cheung, "Adrenomedullin and cardiovascular diseases," JRSM Cardiovascular Disease, vol. 1, no. 5, article 14, 2012.

[8] D. C. Bunton, M. C. Petrie, C. Hillier, F. Johnston, and J. J. V. McMurray, "The clinical relevance of adrenomedullin: a promising profile?” Pharmacology \& Therapeutics, vol. 103, no. 3, pp. 179-201, 2004.

[9] Y.-X. Chen and C.-S. Li, "Prognostic value of adrenomedullin in septic patients in the ED," The American Journal of Emergency Medicine, vol. 31, no. 7, pp. 1017-1021, 2013.

[10] Y.X. Chen and C. S. Li, “The predictive value of adrenomedullin for development of severe sepsis and septic shock in emergency department," BioMed Research International, vol. 2013, Article ID 960101, 6 pages, 2013.

[11] K. Nakao, A. Yasoda, K. Ebihara, K. Hosoda, and M. Mukoyama, "Translational research of novel hormones: lessons from animal models and rare human diseases for common human diseases," Journal of Molecular Medicine, vol. 87, no. 10, pp. 1029-1039, 2009.

[12] S. Hayek and M. Nemer, "Cardiac natriuretic peptides: from basic discovery to clinical practice," Cardiovascular Therapeutics, vol. 29, no. 6, pp. 362-376, 2011.

[13] A. Grzywa-Celiñska, R. Celiñski, K. Kwaśniewska, M. Dyczko, A. Prystupa, and J. Mosiewicz, "The usefulness of natriuretic peptides measurements in the diagnostics of chosen cardiovascular diseases," Polski Merkuriusz Lekarski, vol. 34, no. 202, pp. 232-234, 2013.

[14] K. L. Turner, L. J. Moore, S. R. Todd et al., "Identification of cardiac dysfunction in sepsis with B-type natriuretic peptide," Journal of the American College of Surgeons, vol. 213, no. 1, pp. 139-146, 2011.

[15] R. Witthaut, C. Busch, P. Fraunberger et al., "Plasma atrial natriuretic peptide and brain natriuretic peptide are increased in septic shock: impact of interleukin-6 and sepsis-associated left ventricular dysfunction," Intensive Care Medicine, vol. 29, no. 10, pp. 1696-1702, 2003.

[16] M. Piechota, M. Barylski, S. Hannam, D. P. Mikhailidis, J. Rysz, and M. Banach, "Natriuretic peptides in septic patients," Current Medicinal Chemistry, vol. 16, no. 30, pp. 4020-4031, 2009.

[17] Y. Liu, Q. Li, J.-J. Li, and B. Geng, "The clinical value of atrial natriuretic peptide in early diagnosis of sepsis," Zhonghua Wei Zhong Bing Ji Jiu Yi Xue, vol. 25, no. 11, pp. 669-672, 2013.

[18] M. M. Levy, M. P. Fink, J. C. Marshall et al., "2001 SCCM/ESICM/ACCP/ATS/SIS International Sepsis Definitions Conference," Intensive Care Medicine, vol. 29, no. 4, pp. 530-538, 2003.
[19] M. J. Bader, B. Eisner, F. Porpiglia, G. M. Preminger, and H.G. Tiselius, "Contemporary management of ureteral stones," European Urology, vol. 61, no. 4, pp. 764-772, 2012.

[20] J. R. Eswara, A. Sharif-Tabrizi, and D. Sacco, "Positive stone culture is associated with a higher rate of sepsis after endourological procedures," Urological Research, vol. 41, no. 5, pp. 411414, 2013.

[21] J.-L. Vincent, Y. Sakr, C. L. Sprung et al., "Sepsis in European intensive care units: results of the SOAP study," Critical Care Medicine, vol. 34, no. 2, pp. 344-353, 2006.

[22] F. M. E. Wagenlehner, A. Pilatz, and W. Weidner, "Urosepsisfrom the view of the urologist," International Journal of Antimicrobial Agents, vol. 38, supplement, pp. 51-57, 2011.

[23] B. M. Y. Cheung and F. Tang, "Adrenomedullin: exciting new horizons," Recent Patents on Endocrine, Metabolic \& Immune Drug Discovery, vol. 6, no. 1, pp. 4-17, 2012.

[24] D. L. Hay, C. S. Walker, and D. R. Poyner, "Adrenomedullin and calcitonin gene-related peptide receptors in endocrine-related cancers: opportunities and challenges," Endocrine-Related Cancer, vol. 18, no. 1, pp. C1-C14, 2011.

[25] T. Eto, J. Kato, and K. Kitamura, "Regulation of production and secretion of adrenomedullin in the cardiovascular system," Regulatory Peptides, vol. 112, no. 1-3, pp. 61-69, 2003.

[26] R. Marino, J. Struck, A. S. Maisel, L. Magrini, A. Bergmann, and S. Di Somma, "Plasma adrenomedullin is associated with short-term mortality and vasopressor requirement in patients admitted with sepsis," Critical Care, vol. 18, no. 1, article R34, 2014.

[27] R. Irzmański, L. Pawlicki, M. Charłusz, and J. Kowalski, "Concentration of natriuretic peptides in patients suffering from idiopathic arterial hypertension and left ventricular diastolic dysfunction confirmed by echocardiography," Clinical and Experimental Hypertension, vol. 34, no. 7, pp. 530-540, 2012.

[28] C. Federico, "Natriuretic peptide system and cardiovascular disease," Heart Views, vol. 11, no. 1, pp. 10-15, 2010.

[29] R.-L. Wang and F.-X. Kang, "Prediction about severity and outcome of sepsis by proatrial natriuretic peptide and proadrenomedullin," Chinese Journal of Traumatology, vol. 13, no. 3, pp. 152-157, 2010.

[30] M. Lipinska-Gediga, M. Mierzchala, and G. Durek, "Pro-atrial natriuretic peptide (pro-ANP) level in patients with severe sepsis and septic shock: prognostic and diagnostic significance," Infection, vol. 40, no. 3, pp. 303-309, 2012.

[31] A. S. Omar, M. Ur Rahman, G. S. Dhatt, G. O. Salami, and S. Abuhasna, "Dynamics of brain natriuretic peptide in critically ill patients with severe sepsis and septic shock," Saudi Journal of Anaesthesia, vol. 7, no. 3, pp. 270-276, 2013.

[32] S. M. Perman, A. M. Chang, J. E. Hollander et al., "Relationship between B-type natriuretic peptide and adverse outcome in patients with clinical evidence of sepsis presenting to the emergency department," Academic Emergency Medicine, vol. 18, no. 2, pp. 219-222, 2011.

[33] T. Nakamura, T. Suzuki, Y. Kawagoe, and H. Koide, "Polymyxin B-immobilized fiber hemoperfusion attenuates increased plasma atrial natriuretic Peptide and brain natriuretic peptide levels in patients with septic shock," ASAIO Journal, vol. 54, no. 2, pp. 210-213, 2008.

[34] J. Londoño, A. L. León, F. Rodríguez et al., "Serum lactate in the emergency department as a prognostic factor in patients with sepsis without hypotension," Medicina Clinica, vol. 141, no. 6, pp. 246-251, 2013. 
[35] S. H. Hoeboer, E. Alberts, I. Van Den Hul, A. N. Tacx, Y. J. Debets-Ossenkopp, and J. A. B. Groeneveld, "Old and new biomarkers for predicting high and low risk microbial infection in critically ill patients with new onset fever: a case for procalcitonin," The Journal of Infection, vol. 64, no. 5, pp. 484493, 2012.

[36] C. Pierrakos and J.-L. Vincent, "Sepsis biomarkers: a review," Critical Care, vol. 14, no. 1, article R15, 2010. 


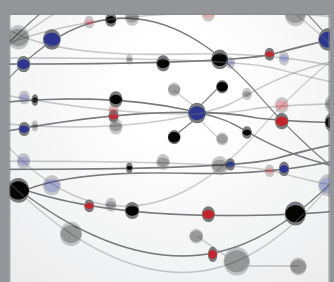

The Scientific World Journal
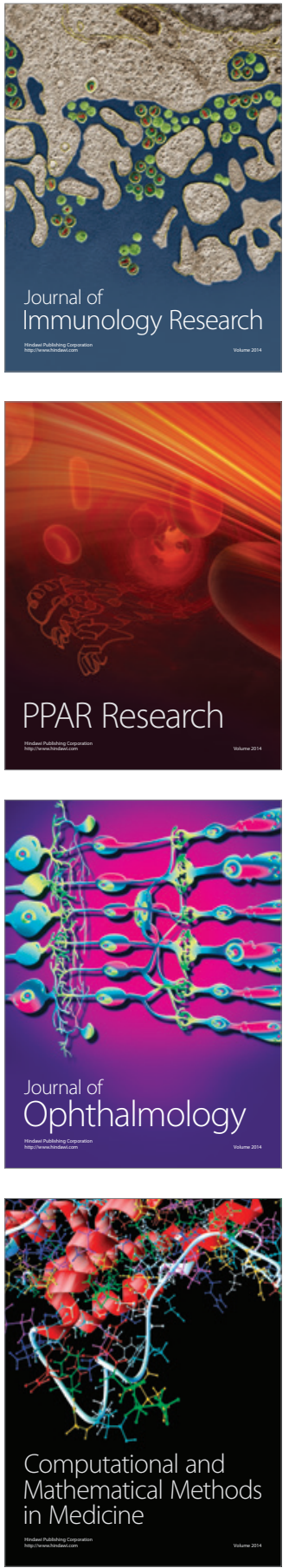

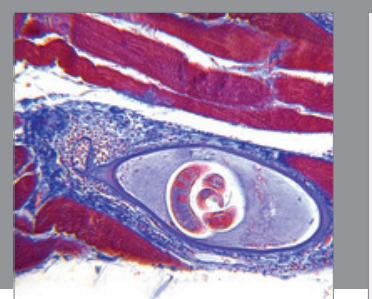

Gastroenterology Research and Practice

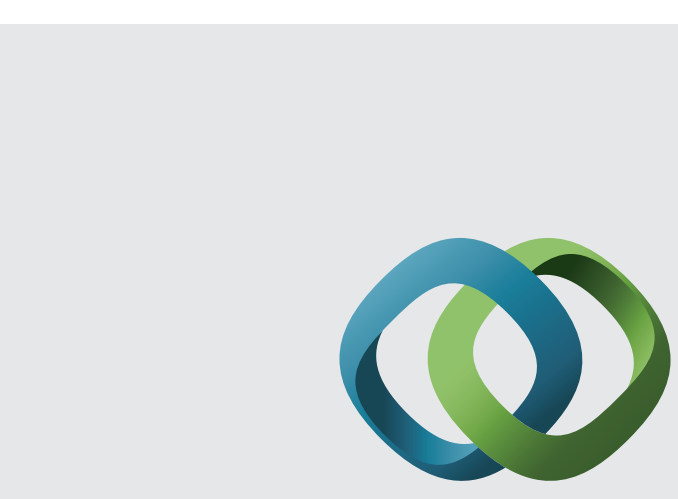

\section{Hindawi}

Submit your manuscripts at

http://www.hindawi.com
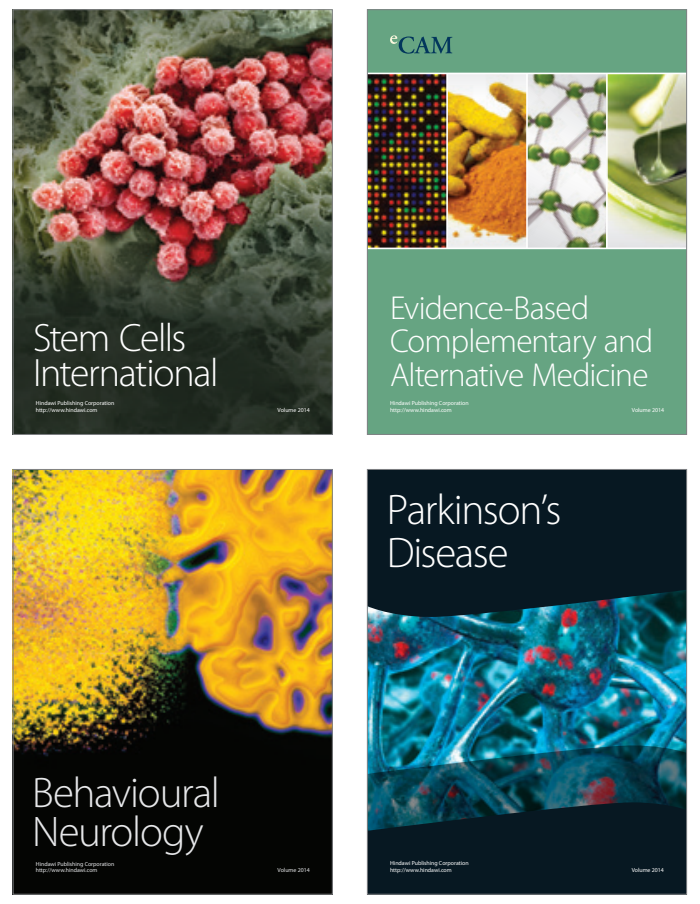
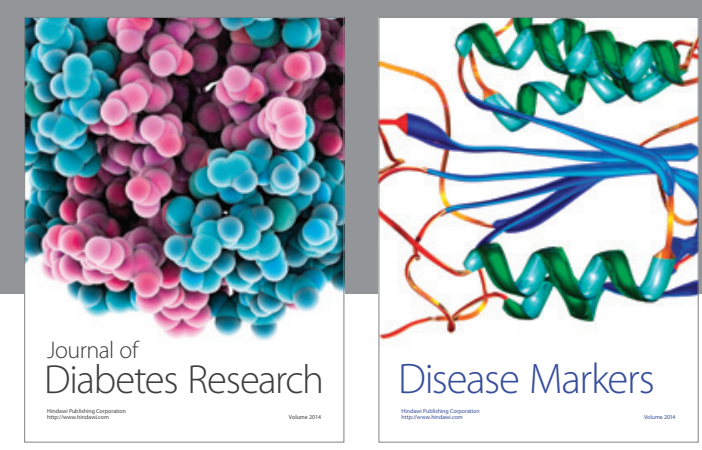

Disease Markers
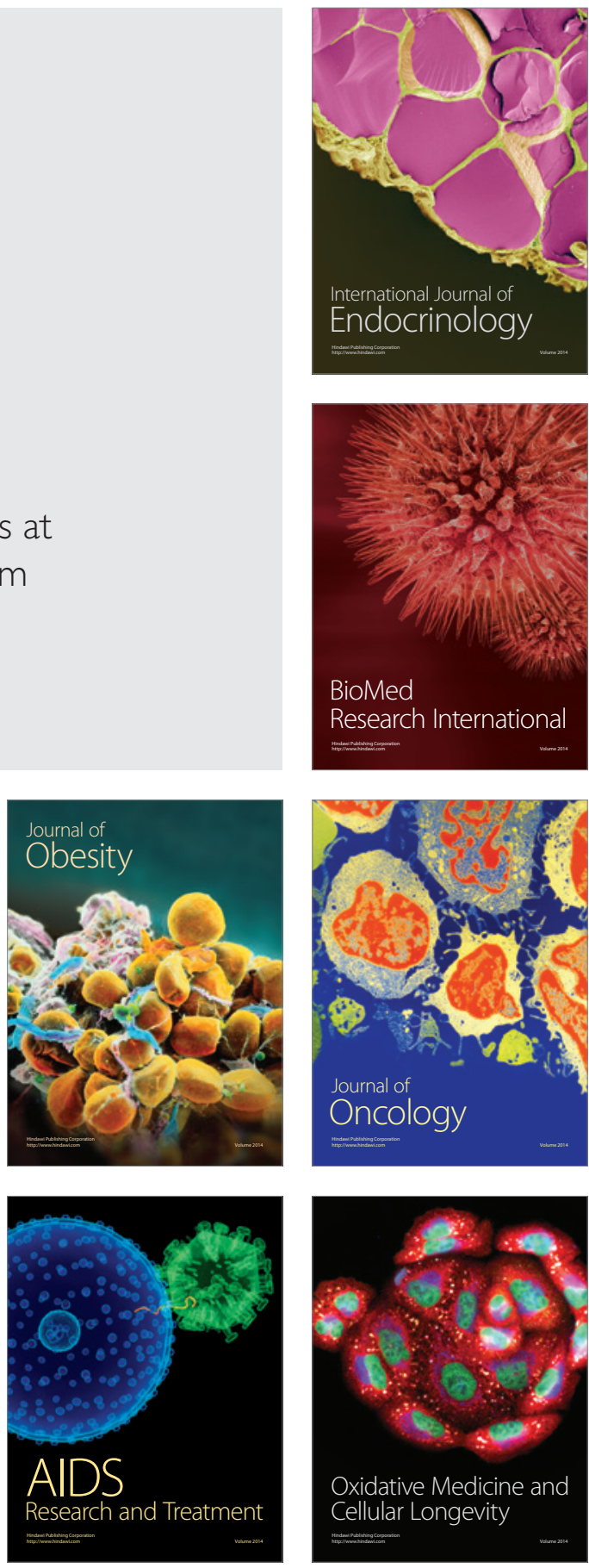